\title{
Nassarius Gibbosulus (Linné 1758 : Buccinum)
}

\section{Marian Vanhaeren et Francesco d'Errico}

\section{OpenEdition}

Journals

Édition électronique

URL : https://journals.openedition.org/encyclopedieberbere/2677

DOI : 10.4000/encyclopedieberbere. 2677

ISSN : 2262-7197

\section{Éditeur}

Peeters Publishers

\section{Édition imprimée}

Date de publication : 4 octobre 2012

Pagination : 5256-5258

ISBN : 978-90-429-2640-0

ISSN : 1015-7344

\section{Référence électronique}

Marian Vanhaeren et Francesco d'Errico, « Nassarius Gibbosulus (Linné 1758 : Buccinum) », Encyclopédie berbère [En ligne], 33 | 2012, document N14, mis en ligne le 23 novembre 2020, consulté le 17 février 2022. URL : http://journals.openedition.org/encyclopedieberbere/2677 ; DOI : https:// doi.org/10.4000/encyclopedieberbere.2677

Ce document a été généré automatiquement le 17 février 2022.

(c) Tous droits réservés 


\title{
Nassarius Gibbosulus (Linné 1758 : Buccinum)
}

\author{
Marian Vanhaeren et Francesco d'Errico
}

Gastéropode marin, également connu sous le synonyme de Arcularia gibbosula (Pallary 1912) ou sous le nom commun de "Nasse bossue ", il peut être confondu avec l'espèce sympatrique Nassarius circumcinctus (Adams A. 1852 : Nassa). Adaptée à des eaux peu profondes à substrat sableux, cette espèce est présente actuellement en Méditerranée orientale et centrale, du Proche Orient au Golfe de Gabès, en Tunisie. Pendant le dernier interglaciaire, elle était également présente sur le littoral nord-africain et sur la côte atlantique du Maroc. Des coquilles de Nassarius gibbosulus sont signalées dans le Quaternaire marin et les plages fossiles à Strombes près de Monastir en Tunisie (d'Errico et al. 2009) et dans les terrasses marines pléistocènes d'Agadir, Sihel, Douar Arhoud, et Dar-es-Soltan au Maroc (Ruhlman 1951, Lecointre 1952, Brébion et al. 1984, Weisrock et al. 1999).

2 Des travaux récents révèlent que ce gastéropode a été utilisé pour façonner les plus anciens objets de parure connus à ce jour. Six sites archéologiques, datés entre 100000 et 70000 ans, ont livré des coquilles Nassarius gibbosulus perforées. Il s'agit de Skhul, en Israël, d'Oued Djebbana, en Algérie et des Grottes des Pigeons (Taforalt), du Rhafas, d'Ifri n'Ammar et des Contrebandiers au Maroc (Vanhaeren et al. 2006, Bouzzougar et al. 2007, d'Errico et al. 2009). Dans le premier site les coquillages sont associés au Moustérien levantin, dans les autres à l'Atérien. Trois de ces sites sont distants de 60 à 40 kilomètres de la mer, ce qui exclut un transport par des agents naturels. L'étude des spécimens découverts à Taforalt a révélé qu'ils ont été collectés morts sur la plage, ce qui élimine l'hypothèse d'un ramassage à but alimentaire. Certaines pièces bien conservées portent encore les marques laissées par les outils en pierre utilisés pour les perforer ou révèlent, à l'échelle microscopique, des usures non présentes à l'état naturel, interprétées par les archéologues comme des traces occasionnées par le port prolongé comme objets de parure. Plusieurs spécimens présentent sur leur surface des traces d'ocre provenant vraisemblablement du lien ou du contact des coquillages avec la peau ou les vêtements ocrés. Les scientifiques ont découvert des coquillages noircis 
par une action de chauffe qui précèderait les traces d'utilisation de ces spécimens. Certains coquillages ont donc pu être chauffés volontairement dans un milieu réducteur en présence de matière organique pour changer leur couleur et ainsi composer des parures de différentes couleurs.

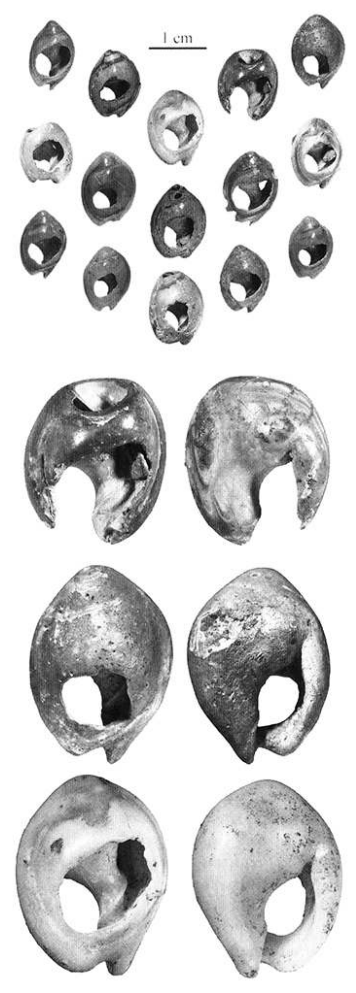

FIgURE. COQUILLES DE NASSARIUS gIBBOSULUS UTILISÉES COMME OBJETS DE PARURE, DÉCOUVERTES DANS LeS COUCHES ATÉRIENNES DE LA GROTTE DES PIgEONS (TAFORALT, MAROC) DATÉES DE 82000 ANS. (PH VANHAEREN/D'ERRICO, FOUILLES BOUZOUggAR/BARTON.)

3 Les coquilles de Nassarius perforées et ocrées apporteraient la preuve d'une culture matérielle symbolique présente il y a 80000 ans, partagée par des populations de la Méditerranée orientale et méridionale. Cette tradition semble disparaître entre 70000 et 30 000. Les coquilles de Nassarius sont à nouveau utilisées comme objets de parure à partir de 30000 ans. On les rencontre par exemple dans les sites Ibéromaurusiens de l'Oued el-Akarit* (Camps-Fabrer 1960) ou dans le Capsien de El-Mekta* (Gobert 1957). Elles sont présentes dans les sites de l'Holocène du nord du Maroc, de l'Algérie et de la Tunisie.

\section{BIBLIOGRAPHIE}

BARTON R.N.E., BOUZOUGGAR A., COLLCUTT S.N., SCHWENNINGER J.-L., CLARK-BALZAN L., 2009 - “OSL dating of the Aterian levels at Dar es-Soltan I (Rabat, Morocco) and possible implications for the dispersai of modem Homo sapiens", Quat. Sci. Rev. 28, p. 1914-1931. 
BOUZOUGgar A., BARTON N., VANHAEREN M., D'ERRICO F., COllCUTT S., HIGHAM T., HODGE E., PARFitT S., RHODES E., SCHWENNINGER J.-L., STRINGER C., TURNER E., WARD S., MOUTMIR A., STAMBOULI A., 2007 "82,000-year-old shell beads from North Africa and implications for the origins of modem human behaviour, Proceedings of the National Academy of Sciences, USA104, p. 9964-9969.

BRÉBION PH., HOANG C.T. ET WEISROCK A., 1984 - « Intérêt des coupes d'Agadir-Port pour l'étude du Pleistocène supérieur marin du Maroc », Bull. Mus. nat. Hist. nat., Pans, 6, p. 129-151.

CAMPS-FABRER H., 1960 - « Parures des temps préhistoriques en Afrique du Nord », Libyca VIII, p. 9-220.

LECOINTRE G., 1952 - «Recherches sur le Néogène et le Quaternaire marin de la côte atlantique du Maroc », Notes et Mém. Serv. géol. Maroc, 99, 2 vol., 198 p. et 173 p.

WEISROCK A., OCCHIETTI S., HOANG CHI-TRACH, LAURIAT-RAGE A., BREBION P., PICHET P. 1999 - « Les séquences littorales pléistocènes de l'Atlas atlantique entre Cap Rhir et Agadir, Maroc ", Quaternaire, vol. 10, $\mathrm{n}^{\circ} 2-3$, p. 227-244.

RUHLMANN A., 1951 - The Prehistoric Cave of Dar-es-Soltan (Hésperis 11), Institut des Hautes Etudes Marocaines, Paris, Larose.

D'ERRICO F., VANHAEREN M., BARTON N., BOUZOUGGAR A., MIENIS H., RICHTER D., HUBlin J.-J., MCPHERRON S.P., LOZOUET P., 2009 - "Additional evidence on the use of personal ornaments in the Middle Paleolithic of North Africa”, Proceedings of the National Academy of Science USA 106, p.16051-16056. VANHAEREN M., D'ERRICO F., STRINGER C., JAMES S.L., TODD J., MIENIS H.K., 2006 - “Middle Palaeolithic Shell Beads in Israël and Algeria", Science 312, p. 1785-1788.

INDEX

Mots-clés : Paléolithique, Parure, Préhistoire, Zoologie 